\title{
Kandori fleck retina
}

INSERM

\section{Source}

INSERM. (1999). Orphanet: an online rare disease and orphan drug data base. Kandori fleck retina. ORPHA:99179

Kandori fleck retina is a rare, genetic retinal dystrophy disorder characterized by irregular, sharply defined, yellowish-white lesions of variable size that are distributed mainly in the nasal equatorial region of the retina, with a tendency to confluence, that are not associated with any vascular or optic nerve abnormalities. They frequently manifest as mild and stationary night blindness. 